University of Nebraska - Lincoln

DigitalCommons@University of Nebraska - Lincoln

Ralph Skomski Publications

Research Papers in Physics and Astronomy

May 2007

\title{
Magnetic impurities in magic-number clusters
}

\author{
Ralph Skomski \\ University of Nebraska-Lincoln, rskomski2@unl.edu \\ David J. Sellmyer \\ University of Nebraska-Lincoln, dsellmyer@unl.edu
}

Follow this and additional works at: https://digitalcommons.unl.edu/physicsskomski

Part of the Physics Commons

Skomski, Ralph and Sellmyer, David J., "Magnetic impurities in magic-number clusters" (2007). Ralph Skomski Publications. 43.

https://digitalcommons.unl.edu/physicsskomski/43

This Article is brought to you for free and open access by the Research Papers in Physics and Astronomy at DigitalCommons@University of Nebraska - Lincoln. It has been accepted for inclusion in Ralph Skomski Publications by an authorized administrator of DigitalCommons@University of Nebraska - Lincoln. 


\title{
Magnetic impurities in magic-number clusters
}

\author{
Ralph Skomski ${ }^{\mathrm{a})}$ and D. J. Sellmyer \\ Department of Physics and Astronomy, University of Nebraska, Lincoln, Nebraska 68588 \\ and Nebraska Center for Materials and Nanoscience, University of Nebraska, Lincoln, Nebraska 68588
}

(Presented on 10 January 2007; received 31 October 2006; accepted 30 January 2007; published online 7 May 2007)

It is investigated how magnetic impurities modify the behavior of metallic clusters. Two complementary models are used, an $s-d$ exchange model with a stable magnetic moment and a Hubbard-type Kondo model. The $s-d$ and $s-f$ interactions are modeled by a pointlike potential, as in the Ruderman-Kittel-Kasuya-Yosida approximation, but the perturbed levels are obtained by diagonalizing the interaction matrix rather than using perturbation theory. The spin polarization of the conduction electrons due to the magnetic impurities leads to a reduction of the highest occupied molecular orbital-lowest unoccupied molecular orbital splitting. A particularly interesting case is Pt, which is used in catalysis and whose well-delocalized $5 d$ electrons are easily spin polarized by conduction electrons. Strikingly, the simplest realization of the Kondo effect is reproduced by an unrestricted Hartree-Fock approximation. (C) 2007 American Institute of Physics.

[DOI: $10.1063 / 1.2713228]$

\section{INTRODUCTION}

It is well known that one-electron states in metallic clusters yield magic-number islands of structural stability. ${ }^{1}$ The stability is given by the number $N$ of electrons ( $N$ $=2,8,18, \ldots)$, with a relatively weak dependence on other atomic parameters and with some dependence on the shape of the cluster. The magic numbers reflect the boundary conditions imposed by the cluster surface and are a mesoscopic quantum-confinement effect similar to the formation of electron shells in atoms. ${ }^{2,3}$ This is different from the stability of electron compounds, such as $\mathrm{Cu}_{5} \mathrm{Zn}_{8},{ }^{4}$ which depends on the electron density and where details of the band structure play an important role.

Due to the relatively large splitting between the highest occupied and the lowest unoccupied molecular orbitals (HOMO-LUMO splitting), these structures are of interest in optics. Dots with magnetic impurities ${ }^{5,6}$ also have potential applications in spintronics, catalysis, and biomedicine. For example, the catalytic activity of particles critically depends on the energy surface, ${ }^{7}$ and magnetic ions may be used to tune the corresponding energy levels.

The behavior of conduction electrons in small metallic clusters is surprisingly well described by the jellium approximation, where the one-electron potential is constant. ${ }^{1}$ This reflects the extended character of the involved electrons and the pseudopotential in the clusters, which ensures that the wave functions are orthogonal to the core states, reducing the density $\psi_{\mathbf{k}}^{*}(\mathbf{r}) \psi_{\mathbf{k}}(\mathbf{k})$ near the atomic cores but leaving the picture otherwise unchanged. Pictorially speaking, atomic cores are almost "transparent" to conduction electrons. ${ }^{4}$ However, magnetic impurities go beyond the jellium model, because their moments are associated with partially filled inner electron shells.

\section{PERTURBED ONE-ELECTRON STATES}

Figure 1 shows the considered geometry, a cubic nanoparticle with a stable magnetic impurity $(S=1 / 2)$. Iron-series transition-metal atoms may lose their moments in a metallic environment, ${ }^{5,6}$ which then requires a different treatment. The present model also applies to tripositive rare-earth ions which have, with the exception of $\mathrm{Ce}^{3+}$, very stable $4 f$ moments. In an $s-d$ interaction picture, the spin-dependent part of the Hamiltonian is $-J \mathbf{s} \cdot \mathbf{S}$, where $\mathbf{s}$ and $\mathbf{S}$ are the extended and localized spins, respectively, and $J \sim \psi_{\mathbf{k}}^{*}(\mathbf{r}) \psi_{\mathbf{k}}(\mathbf{r})$ is the $s-d$ exchange (or spin-dependent potential). Since $\psi_{\mathbf{k}}^{*}(\mathbf{r}) \psi_{\mathbf{k}}(\mathbf{r})$ is proportional to the electron density, $J \sim 1 / N$, where $N$ is the number of atoms per cluster. As we will see in Sec. III, the same dependence is obtained from the Kondo model. The $s-d$ interaction yields a spin splitting of the cluster levels, and the eigenenergies are obtained by diagonalizing the interaction matrix for the 12 lowest-lying energy levels.

Figure 2 shows some low-lying wave functions for undoped [Fig. 2(a)-2(c)] and doped [Fig. 2(d)] clusters. By symmetry, many matrix elements are zero, and the wave functions have a relatively simple structure. Figure 2(d) illustrates how a localized magnetic impurity in the center of the cube affects the ground state by yielding an admixture of excited states. The wave functions depend on the spin orien-

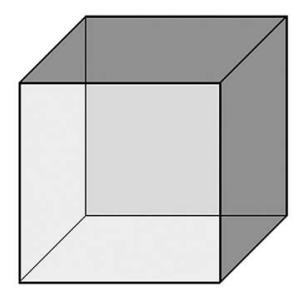

Nonmagnetic Jellium

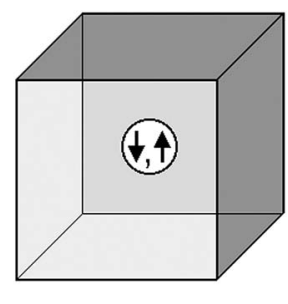

Magnetic Impurity
FIG. 1. Cluster with cubic geometry: (a) nonmagnetic jellium and (b) magnetic impurity. 


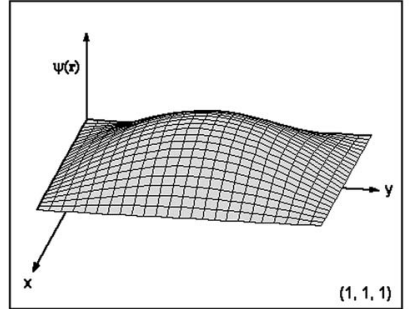

(a)

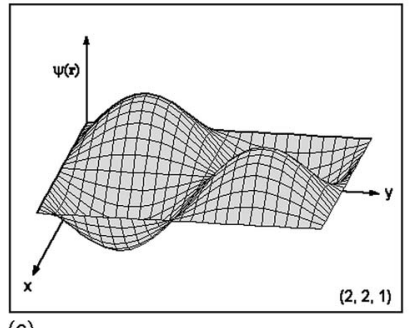

(c)

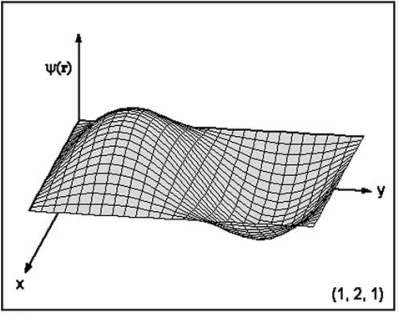

(b)

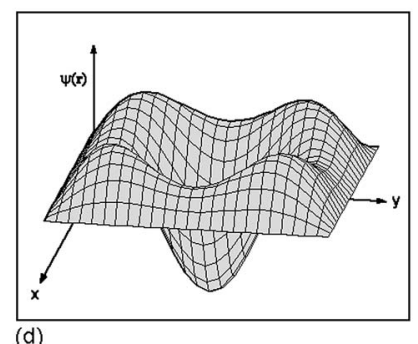

(d) tential of nanoparticles, it is necessary to stabilize the magnetization at the reaction temperature. This may be achieved by using several magnetic impurities per cluster, by a moderate pressure to realize an effective exchange coupling ${ }^{11,12}$ between particles, and/or by using catalytically inert particles such as $\mathrm{Fe}$ as exchange bridges.

\section{KONDO EFFECT}

The jellium model goes beyond the independent-electron approximation, because it generally includes correlations which scales as $n^{-1 / 3},{ }^{10}$ but magnetic impurities complicate the situation. An interesting correlation effect in systems containing both localized and extended electrons is the Kondo effect. In a narrow sense, the term refers to the resistance minimum observed in metals containing magnetic impurities, but the phenomenon also occurs in other correlated systems, such as heavy fermions and manganites. It involves strongly correlated electrons in localized orbitals $|\operatorname{loc}\rangle$ and weakly correlated electrons in extended orbitals $|e x t\rangle$.

In the simplest case, it is sufficient to consider one localized level and one extended level. ${ }^{9}$ In large clusters, many electrons need to be considered, but the finite cluster size yields a long-wavelength cutoff and conserves the essence of the few-electron mechanism. The calculation is similar to that in Ref. 9, but the parameters are obtained for a magnetic impurity coupled to an electron extending over the whole cluster or particle, and we use explicit two-electron wave functions, ${ }^{13}$

$|\psi\rangle=c_{1} \mid$ ext $\rangle \mid$ ext $\rangle+c_{2} \mid$ ext $\rangle \mid$ loc $\rangle+c_{3} \mid$ loc $\rangle \mid$ ext $\rangle+c_{4} \mid$ loc $\rangle \mid$ loc $\rangle$. Note that we consider magnetic nanoparticles for active catalysis, ${ }^{8}$ rather than using them as catalyst supports. An interesting case is magnetic impurities in exchange-enhanced Pauli paramagnets, such as Pt. The wave-vector-dependent susceptibility of these materials ${ }^{9,10}$ contains a long range component which enhances the spin splitting and smoothes the wave function, so that the picture in Fig. 2(d) is no longer restricted to very small particles. To exploit the catalytic po-

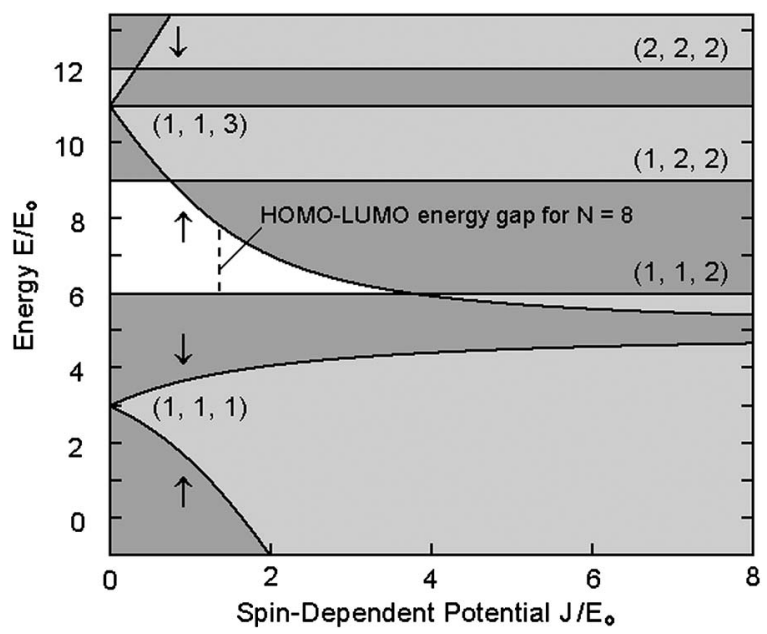

FIG. 3. Energy levels for a cubic quantum dot as a function of the exchange with a magnetic impurity centered in the dot. The number are the quantum numbers $(l, m, n)$ of the levels.
For example, the $c_{2}$ term means that the first and second electrons are in the extended and localized states, respectively. In the basis of Eq. (1), the Hamiltonian is

$$
\mathrm{H}=\left(\begin{array}{cccc}
E_{o} & t & t & 0 \\
t & 0 & 0 & t \\
t & 0 & 0 & t \\
0 & t & t & U-E_{o}
\end{array}\right) .
$$

Here $E_{o}=E_{\text {ext }}-E_{\text {loc }}$ is the orbital energy difference between the extended and localized electrons $\left(E_{o}>0\right), U$ is the Coulomb energy, and $t<0$ is the hopping integral between |ext $\rangle$ and $|l o c\rangle$. The orbital energy $E_{o}$ favors the occupancy of the localized orbital, but the strong Coulomb repulsion $U>E_{o}$ makes the double occupancy of this orbital energetically unfavorable. Since $U$ is very large, we can ignore the admixture of $\mid$ loc $\rangle|\operatorname{loc}\rangle$ character to the system's low-lying states.

In the absence of hopping, the ground state of Eq. (2) is twofold degenerate, corresponding to wave functions $\left|\psi_{ \pm}\right\rangle$ $=\mid$ ext $\rangle \mid$ loc $\rangle \pm \mid$ loc $\rangle \mid$ ext $\rangle$. The symmetric function $\left|\psi_{+}\right\rangle$describes an antiferromagnetic (AFM) spin singlet $(\uparrow \downarrow, S=0)$, whereas the antisymmetric wave function $\left|\psi_{-}\right\rangle$corresponds to a ferromagnetic (FM) triplet ( $\uparrow, S=1)$. In the presence of hopping, the triplet remains unaffected $(E=0)$, but the AFM singlet hybridizes with $\mid$ ext $\rangle|e x t\rangle$. This hybridization lowers the energy of the AFM state by 


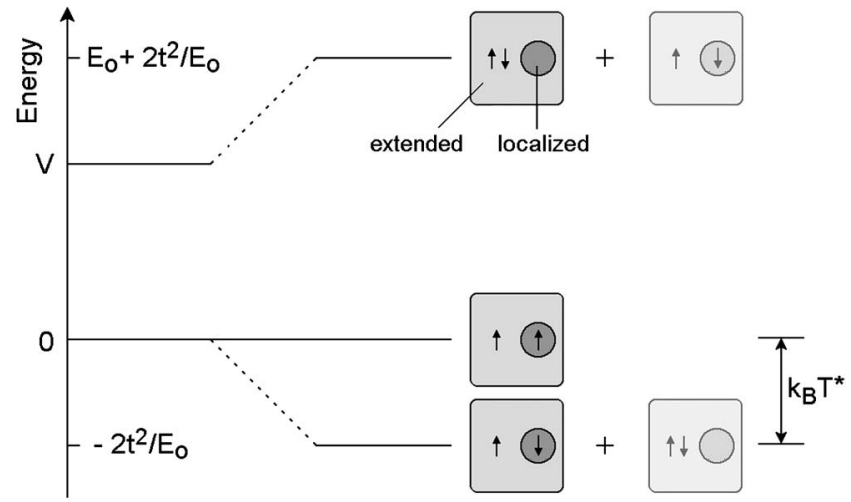

FIG. 4. Energy levels and wave function for an extended quantum state coupled to a localized impurity. $T^{*}$ is the Kondo temperature.

$$
\Delta E=2 t^{2} / E_{o} .
$$

The FM state is unable to benefit from the hopping, because the Pauli principle excludes the double occupancy of any orbital by parallel spins. Figure 4 shows the lowest-lying eigenstates. In the ground state, the impurity spin surrounds itself with a conduction electron of opposite spin orientation. This magnetic screening is a key signature of the Kondo effect.

The Kondo splitting $\Delta E \approx k_{B} T^{*}$ between the $\uparrow \uparrow$ and $\uparrow \downarrow$ levels scales as $1 / N$, because the hopping integral $t$ is essentially the overlap between the delocalized wave function $\mid$ ext $\rangle \sim 1 / N^{1 / 2}$ and the localized wave function. This is in agreement with the simpler interaction picture employed in Sec. II.

It is interesting to note that Eq. (3) is reproduced by an unrestricted Hartree-Fock (HF) approximation. The idea is to use a single Slater determinant constructed from arbitrary one-electron wave functions. An example is Fisher-Coulson wave functions, ${ }^{14}$ which describe the $\mathrm{H}_{2}$ molecule by partially localized one-electron wave functions (see also Ref. 15). By contrast, both bonding and antibonding one-electron wave functions are of the Bloch type, with symmetric electron densities. Here, the FM wave functions are $\left|\psi_{1}\right\rangle=\mid$ ext $\rangle$ and $\left|\psi_{2}\right\rangle=|\operatorname{loc}\rangle$, whereas the AFM state is described by the ansatz $\left|\psi_{1}\right\rangle=\mid$ ext $\rangle$ and $\left|\psi_{2}\right\rangle=\mid$ loc $\rangle+c_{o} \mid$ ext $\rangle$ (Fig. 5). Minimizing the AFM energy with respect to $c_{o}$ yields $c_{o}=-E_{o} / 2 t$ and reproduces Eq. (3). This is striking, because Blochsymmetric and unrestricted Hartree-Fock approximations are a notoriously unreliable tool ${ }^{9}$ to investigate excitations in correlated electron systems. However, in the present case, the relevant first excited state is ferromagnetic, and it is well known that the correlations leave ferromagnetic states unchanged (automatic correlation due to Pauli principle).

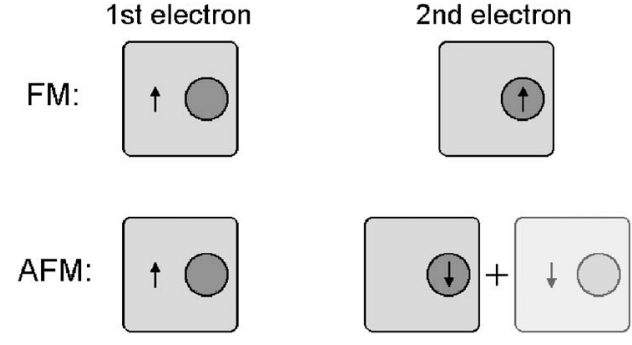

FIG. 5. Wave functions in the unrestricted Hartree-Fock picture. In both the FM and AFM states, one electron is confined to the extended orbital. The second electron is entirely (FM) or largely (AFM) confined to the localized orbital.

\section{CONCLUSIONS}

In summary, we have investigated how magnetic impurities affect extended states in small magnetic clusters. The spin polarization of the conduction electrons leads to a reduction of the HOMO-LUMO splitting, which is unfavorable for optical applications but may be exploited in other applications, such as catalysis. The considered $s$ - $d$ exchange and the Hubbard-type Kondo models yield similar magnetic interaction strength, both scaling as $1 / N$, where $N$ is the number of atoms per cluster. An unusual feature of the considered Hubbard-type interaction model is that the Kondo splitting is reproduced by an unrestricted Hartree-Fock approach.

\section{ACKNOWLEDGMENTS}

This research is supported by NSF MRSEC, DOE, and NCMN

${ }^{1}$ S. M. Reimann, M. Koskinen, H. Häkkinen, P. E. Lindelof, and M. Manninen, Phys. Rev. B 56, 12147 (1997).

${ }^{2}$ X. G. Gong and V. Kumar, Phys. Rev. Lett. 70, 2078 (1993).

${ }^{3}$ J. Li, X. Li, H.-J. Zhai, and L.-Sh. Wang, Science 299, 864 (2003).

${ }^{4}$ A. Cottrell, MRS Bull. 25, 125 (2000).

${ }^{5}$ S. N. Khanna, B. K. Rao, and P. Jena, Phys. Rev. Lett. 89, 016803 (2002).

${ }^{6}$ E. Janssens, S. Neukermans, H. M. T. Nguyen, M. T. Nguyen, and P. Lievens, Phys. Rev. Lett. 94, 113401 (2005).

${ }^{7}$ N. D. Spencer and G. A. Somorjai, Rep. Prog. Phys. 46, 1 (1983).

${ }^{8}$ N. Toshima, Y. Shiraishi, A. Shiotsuki, D. Ikenaga, and Y. Wang, Eur. Phys. J. D 16, 209 (2001).

${ }^{9} \mathrm{P}$. Fulde, Electron Correlations in Molecules and Solids (Springer, Berlin, 1991).

${ }^{10}$ W. Jones and N. H. March, Theoretical Solid State Physics I (Wiley, London, 1973).

${ }^{11}$ R. Skomski, H. Zeng, and D. J. Sellmyer, IEEE Trans. Magn. 37, 2549 (2001).

${ }^{12}$ R. Skomski, J. Phys.: Condens. Matter 15, R841 (2003).

${ }^{13}$ R. Skomski and J. M. D. Coey, Permanent Magnetism (Institute of Physics, Bristol, 1999).

${ }^{14}$ C. A. Coulson and I. Fischer, Philos. Mag. 40, 386 (1949).

${ }^{15}$ L. M. Falicov and R. A. Harris, J. Chem. Phys. 51, 3153 (1969). 